\title{
Extended ammonia observations towards the integral-shaped filament $^{\star}$
}

\author{
Gang $\mathrm{Wu}^{1,2,3,4}$, Keping Qiu ${ }^{2,5}$, Jarken Esimbek ${ }^{1,3}$, Xingwu Zheng ${ }^{2}$, Christian Henkel ${ }^{6,7}$, \\ Dalei $\mathrm{Li}^{1}$, and XiaoHong Han ${ }^{1}$

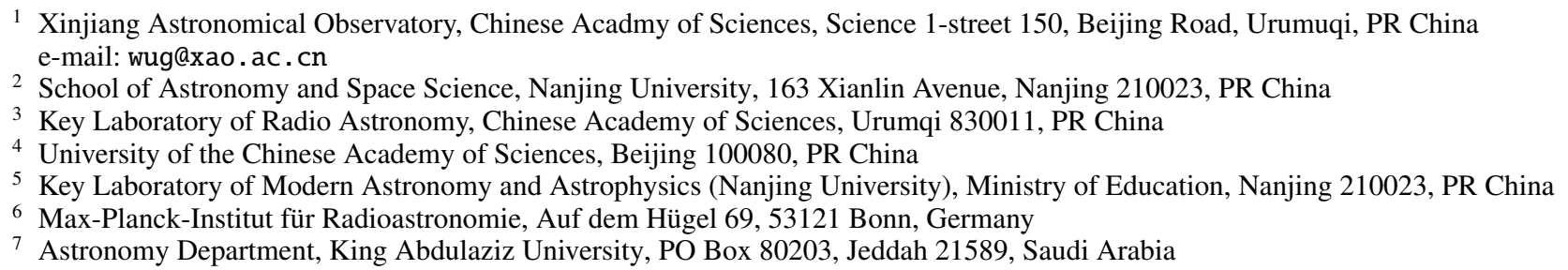

Received 22 December 2016 / Accepted 25 April 2018

\begin{abstract}
Context. Recent observations suggest a scenario in which filamentary structures in the interstellar medium represent the first step towards clumps/cores and eventually star formation. The densest filaments would then fragment into prestellar cores owing to gravitational instability.

Aims. We seek to understand the roles filamentary structures play in high-mass star formation.

Methods. We mapped the integral-shaped filament (ISF) located at the northern end of the Orion A molecular cloud in $\mathrm{NH}_{3}(1,1)$ and $(2,2)$. The observations were made using the $25 \mathrm{~m}$ radio telescope operated by the Xinjiang Astronomical Observatory, Chinese Academy of Sciences. The whole filamentary structure, about $1.2^{\circ} \times 0.6^{\circ}$, is uniformly and fully sampled. We investigate the morphology, fragmentation, kinematics, and temperature properties in this region.

Results. We find that the morphology revealed by the map of velocity-integrated intensity of the $\mathrm{NH}_{3}(1,1)$ line is closely associated with the dust ridge revealed by the Herschel Space Observatory. We identify 6 "lumps" related to the well known OMC-1 to 5 and 11 "sub-clumps" within the map. The clumps and sub-clumps are separated not randomly but in roughly equal intervals along the ISF. The average spacing of clumps is $11.30^{\prime} \pm 1.31^{\prime}(1.36 \pm 0.16 \mathrm{pc})$ and the average spacing of sub-clumps is $7.18^{\prime} \pm 1.19^{\prime}(0.86 \pm 0.14 \mathrm{pc})$. These spacings agree well with the predicted values of the thermal $(0.86 \mathrm{pc})$ and turbulent sausage instability $(1.43 \mathrm{pc})$ by adopting a cylindric geometry of the ISF with an inclination of $60^{\circ}$ with respect to the line of sight. We also find a velocity gradient of about $0.6 \mathrm{~km} \mathrm{~s}^{-1} \mathrm{pc}^{-1}$ that runs along the ISF which likely arises from an overall rotation of the Orion A molecular cloud. The inferred ratio between rotational and gravitational energy is well below unity. Furthermore, fluctuations are seen in the centroid velocity diagram along the ISF. The OMC-1 to 5 clouds are located close to the local extrema of the fluctuations, which suggests that there exist gas flows associated with these clumps in the ISF. The derived $\mathrm{NH}_{3}(1,1)$ and $(2,2)$ rotation temperatures in the OMC-1 are about $30-40 \mathrm{~K}$ while lower temperatures (below $20 \mathrm{~K}$ ) are obtained in the northern and southern parts of the ISF. In OMC-2, OMC-3, and the northern part of OMC-4, we find higher and lower temperatures at the boundaries and in the interior, respectively.
\end{abstract}

Key words. ISM: clouds - ISM: structure - ISM: kinematics and dynamics - stars: formation - ISM: individual objects: ISF

\section{Introduction}

In spite of the crucial role which high-mass stars $\left(>8 M_{\odot}\right)$ play in the evolution of the Universe, their formation and early evolution remains poorly understood (Zinnecker \& Yorke 2007; Tan et al. 2014). High-mass stars always form in stellar clusters embedded in giant molecular clouds (GMCs). Recent observational studies reveal filamentary structures to be omnipresent in molecular clouds (e.g., André et al. 2010; Jackson et al. 2010; Li et al. 2014). Moreover, according to a "globular-filament" scenario of star formation (Schneider \& Elmegreen 1979), filamentary structures in the interstellar medium represent the first step towards precluster clumps/prestellar cores and eventually star forma-

* The reduced data cubes are only available at the CDS via anonymous ftp to cdsarc.u-strasbg.fr $(130.79 .128 .5)$ or via http: //cdsarc.u-strasbg.fr/viz-bin/qcat?]/A+A/616/A111 tion (André et al. 2014). The densest filaments would then fragment into clumps/cores owing to gravitational instability (e.g., Jackson et al. 2010).

Theories and models for filaments or gas cylinders have been studied for more than six decades (e.g., Chandrasekhar \& Fermi 1953; Ostriker 1964; Inutsuka \& Miyama 1992). The instability of gas cylinders shows some differences with respect to threedimensional (3D) Jeans collapse. In a spherical gravitational Jeans collapse, perturbations of all wavenumbers grow at the same rate. However, in gas cylinders, perturbations of certain wavenumbers grow faster than others (Chandrasekhar \& Fermi 1953; Jackson et al. 2010). Therefore, overdensities will tend to form at a length scale corresponding to this fastest growing wavenumber. Observationally, overdensities along filaments should be found at roughly regular intervals (the "sausage" instability; e.g., Jackson et al. 2010). Filaments are promising sites to study and test the physics of molecular cloud (MC) formation and 
fragmentation and to evaluate the earliest stages of high-mass star formation.

The Orion complex is the nearest and probably best studied on-going star formation region that continues to produce both low- and high-mass stars (Bally 2008). At a distance of about $414 \pm 7$ pc (Menten et al. 2007), it can be observed with good linear resolution even with a radio telescope of modest size. Meanwhile it is located about 15 degrees below the Galactic plane, leading to a less confused background than that typically encountered along the Galactic plane. Orion A is the largest MC ( 31.5 $\left.\mathrm{deg}^{2}\right)$ in the Orion complex (Wilson et al. 2005). The integral-shaped filament (ISF) is the compact ridge at the northern end of the cometary Orion A cloud (Bally et al. 1987; Johnstone \& Bally 1999). The ISF contains several clumps, namely OMC-1, OMC-2, OMC-3, OMC-4, and OMC-5 (e.g., Johnstone \& Bally 1999, 2006). The ISF is an active high-mass star formation site and has spawned a sequence of stellar groups of different ages in the past few million years and likely contains hundreds of young stellar objects (Bally 2008).

To obtain physical information from the MCs, ammonia $\left(\mathrm{NH}_{3}\right)$ inversion transitions are an invaluable spectroscopic tool due to the molecule's lack of depletion, its hyperfine structure, and its sensitivity to kinetic temperature (Ho \& Townes 1983). $\mathrm{NH}_{3}(1,1)$ and $(2,2)$ have been proved to be an excellent thermometer under $20 \mathrm{~K}$. It can also be a good thermometer for higher temperatures after some modification but with a reduction in precision (Walmsley \& Ungerechts 1983; Danby et al. 1988; Tafalla et al. 2004). Moreover, the critical densities of $\mathrm{NH}_{3}(1,1)$ and $(2,2)$ are about $10^{3} \mathrm{~cm}^{-3}$ (Evans 1999; Shirley 2015), thus providing a proper tracer for the dense part of the filaments and their fragmentation.

In this paper, we therefore present uniform and fully sampled maps of the ISF in $\mathrm{NH}_{3}(1,1)$ and $(2,2)$ and discuss the morphology, fragmentation, kinematics, and temperature properties in this region.

\section{Observations and database archives}

\section{1. $\mathrm{NH}_{3}$ observations}

The $25 \mathrm{~m}$ radio telescope, operated by the Xinjiang Astronomical Observatory, Chinese Academy of Sciences, was used for all the $\mathrm{NH}_{3}(1,1)$ and $(2,2)$ observations presented here. The rest frequency was set at $23.708564 \mathrm{GHz}$ for observing $\mathrm{NH}_{3}$ $(1,1)$ at $23.694495 \mathrm{GHz}$ and $\mathrm{NH}_{3}(2,2)$ at $23.722633 \mathrm{GHz}$, simultaneously. At this frequency, the telescope has a primary beam width (FWHM) of $125^{\prime \prime}$ obtained from point-like continuum calibrators and a velocity resolution of $0.098 \mathrm{~km} \mathrm{~s}^{-1}$ provided by an 8192 channel Digital Filter Bank in a $64 \mathrm{MHz}$ bandwidth mode. All line intensities reported here are in units of main beam brightness temperature $T_{\mathrm{mb}}$. $T_{\mathrm{A}}^{*}$ values were calibrated against periodically $(6 \mathrm{~s})$ injected signals from a noise diode. Due to a hardware problem, we additionally used GBT (Green Bank Telescope) $\mathrm{NH}_{3}(1,1)$ data (Friesen et al. 2017) to calibrate the spectra to the $T_{\mathrm{mb}}$ scale with $\sim 14 \%$ accuracy; see Appendix A.

The telescope pointing and tracking accuracy is better than $18^{\prime \prime}$. A 22-24.2 GHz dual polarization channel superheterodyne receiver was used as frontend. On a $T_{\mathrm{A}}^{*}$ scale, the typical system temperature is about $50 \mathrm{~K}$ at $23 \mathrm{GHz}$. The map was made using the on-the-fly (OTF) mode with $6^{\prime} \times 6^{\prime}$ grid size and $30^{\prime \prime}$ sample step. All the observations were carried out in January 2014 under excellent weather conditions and above an elevation of $20^{\circ}$.

\subsection{Archival data}

The Herschel Space Observatory's $100 \mu \mathrm{m}, 250 \mu \mathrm{m}$, and $500 \mu \mathrm{m}$ images using SPIRE/PACS parallel scan mode data were taken by Stutz \& Kainulainen (2015). The PACS data and SPIRE data used here are level 2.5 products produced by the Herschel interactive processing environment (HIPE) software, version 12.1 (Ott 2010). The Montage ${ }^{1}$ and APLpy toolkits were used for cutout and plotting.

The archival ${ }^{13} \mathrm{CO}$ spectra were observed with the IRAM $30 \mathrm{~m}$ telescope at Pico Veleta, Spain, in March, April, and October 2008. The data were obtained with the HEterodyne Receiver Array (HERA, 9 dual polarisation pixels arranged in the form of a $3 \times 3$ array with $24^{\prime \prime}$ spacing) with an individual beam width of about $11^{\prime \prime}$. The main beam efficiency at $220.4 \mathrm{GHz}$ was 0.545 . The VErsatile Spectrometric and Polarimetric Array (VESPA) was used as backend providing $320 \mathrm{kHz}$ wide channels, which corresponds to roughly $0.4 \mathrm{~km} \mathrm{~s}^{-1}$. The map was made in the on-the-fly (OTF) mode, with $5^{\prime \prime}$ data sampling in right ascension, and with steps of $12^{\prime \prime}$ in declination. The rms per channel is about $0.2 \mathrm{~K}$ on a main beam brightness temperature scale (for more details, see Berné et al. 2014).

\section{Data reduction and results}

The CLASS and GREG packages of GILDAS ${ }^{2}$, and also python plot packages matplotlib (Hunter 2007) and APLpy ${ }^{3}$ were used for all the data reduction. Second-order polynomial fitting is used for baseline removal and the mean rms per channel is about $0.3 \mathrm{~K}$ on a main beam brightness temperature scale. In order to convert hyperfine blended line widths to intrinsic line widths in the $\mathrm{NH}_{3}$ inversion spectrum (e.g., Barranco \& Goodman 1998), we fitted the averaged spectrum using the GILDAS built-in " $\mathrm{NH}_{3}(1,1)$ " fitting method which can fit all 18 hyperfine components simultaneously.

The $\mathrm{NH}_{3}(1,1)$ and $(2,2)$ velocity-integrated line intensity (zeroth moment) maps are presented as gradations of gray-scale intensities and contours in Fig. 1. The integration range is from 6.5 to $12.5 \mathrm{~km} \mathrm{~s}^{-1}$ to cover the main group of hyperfine components $\left(\Delta F=0\right.$, Ho \& Townes 1983) of the $\mathrm{NH}_{3}(1,1)$ or $(2,2)$ transitions. We adopt this integration range since in relatively diffuse parts of the ISF, where $\mathrm{NH}_{3}$ is barely detectable, the satellite lines $(\Delta F= \pm 1$, Ho \& Townes 1983) are usually not accessible and the velocity integrated intensity including satellite lines would lead to lower signal-to-noise ratios (S/Ns). Meanwhile, we also constructed a velocity integrated intensity image including all hyperfine components (the main group and the four groups of satellite lines). The morphology, especially in the denser regions, is similar to the image only including the main line. Furthermore, the results related to clump and sub-clump identification (Sect. 4.1) are not changed.

In Fig. 1, contours start at $0.96 \mathrm{~K} \mathrm{~km} \mathrm{~s}^{-1}$ ( $4 \sigma$, thick gray line) and go up in steps of $0.96 \mathrm{~K} \mathrm{~km} \mathrm{~s}^{-1}$ (thin gray lines). $\sigma$ is equal to rms $\times \Delta V \times \sqrt{N \_c h a n n e l s}$, where rms is the averaged baseline channel rms of all spectra exported from the baseline fitting with

\footnotetext{
1 This research made use of Montage. It is funded by the National Science Foundation under Grant Number ACI-1440620, and was previously funded by the National Aeronautics and Space Administration's Earth Science Technology Office, Computation Technologies Project, under Cooperative Agreement Number NCC5-626 between NASA and the California Institute of Technology.

2 http://www . iram. fr/IRAMFR/GILDAS/

3 http://aplpy.github.com
} 

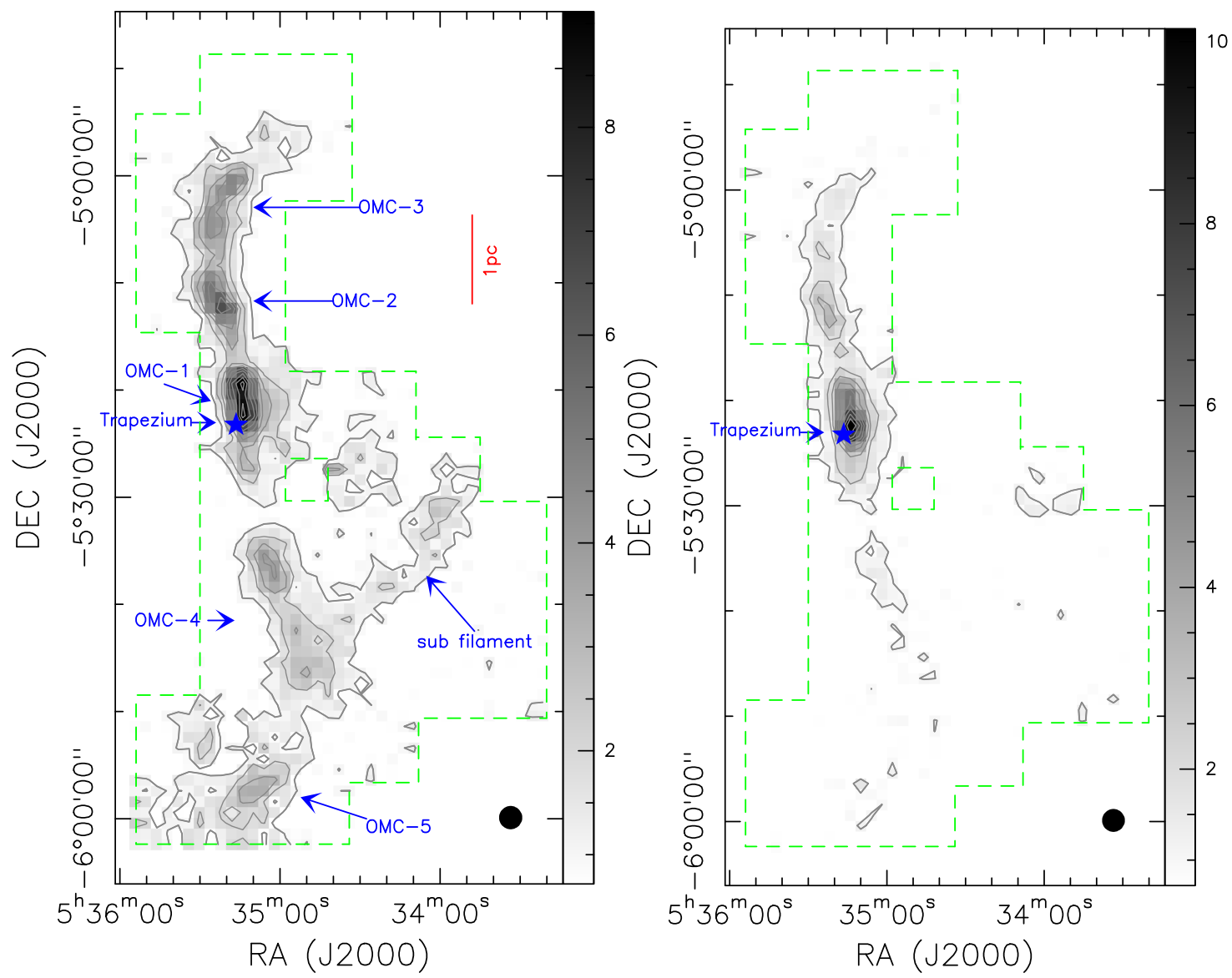

Fig. 1. Integrated intensity (zeroth moment) maps of $\mathrm{NH}_{3}(1,1)($ left $)$ and $(2,2)$ (right). The integration range of each panel is $6.5<V L S R<$ $12.5 \mathrm{~km} \mathrm{~s}^{-1}$. Contours start at $0.96 \mathrm{~K} \mathrm{~km} \mathrm{~s}^{-1}(4 \sigma)$ on a main beam brightness temperature scale and go up in steps of $0.96 \mathrm{~K} \mathrm{~km} \mathrm{~s}^{-1}$. The limits of the mapped region are indicated with green dashed lines. A blue star in each panel indicates the Trapezium cluster and black filled circles in the lower right illustrate the half-power beam size.

CLASS (being part of the GILDAS package), $\Delta V$ is the channel spacing, and $N$ _channels is the channel number in the integrated velocity range $\left(6.5-12.5 \mathrm{~km} \mathrm{~s}^{-1}\right)$. In each panel, the Trapezium cluster is labeled as a blue star and the half-power beam width is illustrated as a black filled circle in the lower right bottom. The limits of the mapped region are indicated with green dashed lines. The clumps OMC- 1 to 5 are labeled in the left panel. The red line in the top right of the left panel illustrates the $1 \mathrm{pc}$ scale at a distance of 414 pc (Menten et al. 2007). An additional "sub filament" structure in the west of OMC-4 is also labeled in the left panel. The whole map consists of a total of 45240 raw spectra. After smoothing to $1^{\prime} \times 1^{\prime}$ areas, 1764 uniformly spaced spectra remain, covering a region about 1.2 degrees in declination and about 0.6 degrees in right ascension.

In the left panel of Fig. 1, the zeroth moment map of $\mathrm{NH}_{3}$ $(1,1)$ shows an integral-shaped morphology $\left(\int\right)$ as observed in ${ }^{13} \mathrm{CO}$ (see Bally et al. 1987). Compared to ${ }^{13} \mathrm{CO}, \mathrm{NH}_{3}(1$, 1) highlights the main filamentary structure and the fragmentation in a clearer way and we find, unlike what is seen in ${ }^{13} \mathrm{CO}$, little diffuse gas around the ISF. In $\mathrm{NH}_{3}(1,1)$, the length of the ISF is about $7 \mathrm{pc}$, and the width is about $1 \mathrm{pc}$ at a distance of $414 \mathrm{pc}$. The densest regions all show elongated morphologies, mainly along the main axis of the ISF, hinting at the existence of sub-structures which are displayed in more detail in Fig. 7 of Friesen et al. (2017). A separated emission region located between the OMC-1 and the already mentioned western sub-filament is not analysed in this work, since only a few pixels show $\mathrm{NH}_{3}(1,1)$ detections in this region. According to the right panel of Fig. 1, most of the $\mathrm{NH}_{3}(2,2)$ line detections are obtained in the northern part of the ISF. Considering weaker $\mathrm{NH}_{3}$ $(1,1)$ intensities in the southern part of the ISF (left panel of Fig. 1) and keeping in mind that $\mathrm{CO}$ is also weaker in the south (e.g., Bally et al. 1987; Wilson et al. 2005), this is likely caused by lower $\mathrm{NH}_{3}$ column densities in the southern part of the ISF.

\section{Discussion}

\subsection{Ammonia morphology and two level fragmentation in the ISF}

A false color Herschel infrared image of the ISF is shown in the left panel of Fig. 2 (red for $500 \mu \mathrm{m}$, green for $250 \mu \mathrm{m}$, and blue for $100 \mu \mathrm{m})$, overlaid with $\mathrm{NH}_{3}(1,1)$ integrated intensity contours as in Fig. 1. From this panel, we can see the contours are closely associated with the dust ridge presented by the Herschel infrared emission. As mentioned in Sect. 3, there is a subfilament in addition to the main filamentary structure, which is extruded in the west of OMC-4 and which is identified for the first time in $\mathrm{NH}_{3}$. The sub-filament has a length of about $20^{\prime}$, that is, about $2 \mathrm{pc}$ at a distance of $414 \mathrm{pc}$. In the left panel of Fig. 2, there is also an obvious infrared feature corresponding to this sub-filament. 

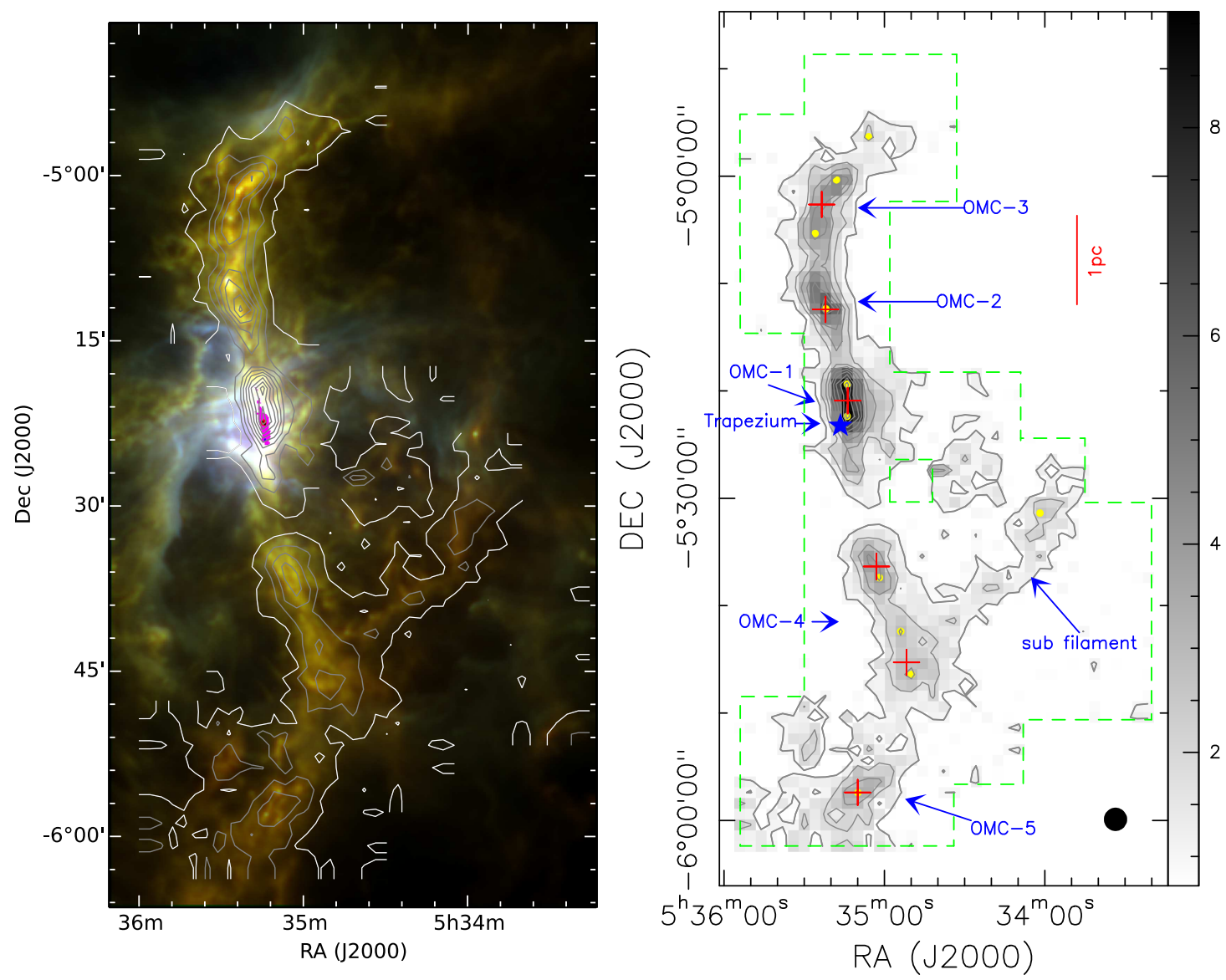

Fig. 2. Left panel: false color image of the ISF (red for $500 \mu \mathrm{m}$, green for $250 \mu \mathrm{m}$, and blue for $100 \mu \mathrm{m}$, all Herschel data) overlaid with $\mathrm{NH}_{3}(1,1)$ integrated intensity contours, as in Fig. 1. Right panel: "clumps" and "sub-clumps" are labeled as red crosses and yellow filled circles, respectively, on the $\mathrm{NH}_{3}(1,1)$ integrated intensity map.

From the $\mathrm{NH}_{3}(1,1)$ integrated intensity map, we find that the six regions with strongest $\mathrm{NH}_{3}$ emission are located along the ISF. In the right panel of Fig. 2, we marked the six regions (two related to OMC-4) with strongest $\mathrm{NH}_{3}$ emission as "clumps" with red crosses on the $\mathrm{NH}_{3}$ map. The emission peaks correspond to significant star-forming regions within the Orion A Molecular Cloud (e.g., Bally et al. 1987; Johnstone \& Bally 1999, 2006). Following the terminology of Johnstone \& Bally (1999, 2006), we name them OMC-1 to 5 . Since the two regions in OMC-4 are separated by more than a typical clump size of $1 \mathrm{pc}$ (e.g., hierarchical fragmentation in Zhang et al. 2009; Wang et al. 2014), we consider them as two separate "clumps". These clumps are not randomly spaced but at roughly equal intervals. The average spacing of these five clumps is $11.30^{\prime} \pm 1.31^{\prime}$ (given uncertainties are errors of the mean), that is, $1.36 \pm 0.16 \mathrm{pc}$ at a distance of $414 \mathrm{pc}$ (Menten et al. 2007). We then used the Clumpfind2d algorithm (Williams et al. 1994) with a threshold of $5 \sigma$ and increments of $2 \sigma$ ( $\sigma$ is defined in Sect. 2.1) to identify sub-structure. Lu et al. (2014) and Ohashi et al. (2016), for example, used similar parameters to identify substructures. Eleven such sub-structures were identified within the $\mathrm{NH}_{3}$ map after excluding a fake one located at the boundary of the map. We also present the pixels used to define these substructures in Appendix B. As the sub-structures are smaller than $1 \mathrm{pc}$ and larger than $0.1 \mathrm{pc}$, we name these sub-structures "subclumps". Pineda et al. (2009) argued the specific selection of the parameters in the Clumpfind2d algorithm may result in slight differences in identifying the condensations, but it works well for relatively isolated sub-clumps which we find in the ISF. In our observation, the clumpfind2d algorithm is more sensitive to the threshold we have chosen, and not to the increments. Using a threshold of $3 \sigma$, the procedure tends to find some additional artificial condensations at the edge of the image. Therefore, we adopt a robust threshold of $5 \sigma$ and increments of $2 \sigma$ leading to an identification of those sub-clumps that one would tend to select by eye. We marked these sub-clumps as yellow filled circles in the right panel of Fig. 2. As we can see, the ten sub-clumps within the main structure of the ISF are also regularly separated. The average spacing is $7.18^{\prime} \pm 1.19^{\prime}$, which corresponds to $0.86 \pm 0.14 \mathrm{pc}$ at $414 \mathrm{pc}$.

The results may suffer from the effect of the small number statistics. Before we interpret the results, we considered 1 million tests of six-element random arrays in the range of 0 to $1.36 \times 6 \mathrm{pc}$. About $1.1 \%$ of these random arrays are more equally spaced (the ratio of the standard deviation of the mean to the mean value is less than $0.16 \mathrm{pc} / 1.36 \mathrm{pc}$ ). Subsequently, we considered 1 million tests of ten-element random arrays in the range of $0-0.86 \times 10 \mathrm{pc}$. About $2.5 \%$ of these random arrays are more equally spaced (the ratio of the standard deviation of the mean to the mean value is less than $0.14 \mathrm{pc} / 0.86 \mathrm{pc}$ ).

The approximately equal intervals of the clumps can be explained by a so-called sausage instability firstly proposed by Chandrasekhar \& Fermi (1953) for an incompressible gas cylinder. Following Jackson et al. (2010), for an isothermal cylinder with infinite radius, the characteristic length between adjacent condensations is about $22 \mathrm{H}$. $\mathrm{H}$ is the isothermal scale height 
given by $H=c_{\mathrm{s}}\left(4 \pi G \rho_{\mathrm{c}}\right)^{-1 / 2}$, where $c_{\mathrm{s}}$ is the sound speed, $G$ is the gravitational constant, and $\rho_{\mathrm{c}}$ is the gas mass density at the center of the filament. For an isothermal cylinder of finite radius $R$ surrounded by an external, uniform medium, the characteristic length for $R \gg H$ is also about $22 H$. If $R \ll H$, the characteristic length becomes about $11 R$.

We assumed the ISF is an isothermal cylinder with a typical kinetic temperature of $18 \mathrm{~K}$ (see Sect. 4.3) and a central volume average density of the ISF of $10^{4} \mathrm{~cm}^{-3}$ (Bally et al. 1987; Cesaroni \& Wilson 1994; Johnstone \& Bally 1999). Then the sound speed $c_{\mathrm{s}}=\sqrt{k T / \mu_{H} m_{H}}=0.25 \mathrm{~km} \mathrm{~s}^{-1}$, taking $\mu_{H}=2.4$ and $m_{H}=1.674 \times 10^{-24} \mathrm{~g}$. The isothermal scale height is $H \sim 0.04 \mathrm{pc}$. Therefore, under the regime $R \gg H$, the characteristic length $(22 H)$ of the ISF is about $1 \mathrm{pc}$. Assuming a median inclination of a randomly oriented filament of $60^{\circ}$ with respect to the line of sight (Genzel \& Stutzki 1989; Hanawa et al. 1993), the projected spacing will be $1 \mathrm{pc} \times \sin \left(60^{\circ}\right) \sim 0.86 \mathrm{pc}$, which is exactly the averaged spacing of the sub-clumps $(0.86 \pm 0.14 \mathrm{pc})$. As we can see, the inclination of the ISFis relevant in defining the separation of the clumps. We are collecting distance measurements in the entire Orion A filament to fit the inclination of the ISF in a separate paper. According to the preliminary result of the fitting of 17 YSO distances derived by the VLBA paradox measurement (Kounkel et al. 2017), the derived inclination is about $60^{\circ}$ and the northern part is more nearby (Wu et al., in prep.). As suggested by Jackson et al. (2010), the above calculations assume that thermal pressure dominates the overall pressure budget. Because the observed line widths in most molecular clouds typically exceed the thermal sound speed, turbulent pressure usually dominates over thermal pressure. To consider the turbulence's contribution, we have to replace the sound speed by the typically observed velocity dispersion $\sigma$. Therefore, adopting a FWHM line width of $1.0 \mathrm{~km} \mathrm{~s}^{-1}$ for $\mathrm{NH}_{3}(1,1)$ (see Sect. 4.2), the velocity dispersion $\sigma$ is related to the FWHM line width $\Delta V$ as $\sigma=\Delta V / \sqrt{8 \ln (2)}=0.42 \mathrm{~km} \mathrm{~s}^{-1}$ for a Gaussian line profile. Then the effective scale height, replacing the previously defined $H$-parameter, is about $0.07 \mathrm{pc}$. The characteristic length then becomes about $1.65 \mathrm{pc}$. Assuming again that the inclination of the ISF is $60^{\circ}$, the projected spacing will be $1.65 \mathrm{pc} \times \sin \left(60^{\circ}\right)$ $\sim 1.43 \mathrm{pc}$, which is in good agreement with the averaged separation of the clumps we find $(1.36 \pm 0.16 \mathrm{pc})$. In short, we observe a two-level fragmentation in the ISF, which can be explained by thermal and turbulent sausage instability, respectively. In this context it is important to note that the Jeans length $\lambda_{\mathrm{J}} \sim 0.27 \mathrm{pc}(T=18 \mathrm{~K}$, $\left.n\left(H_{2}\right)=10^{4} \mathrm{~cm}^{-3}\right)$ cannot explain this fragmentation.

The previous spacing calculations are all based on the typical temperature and velocity, which represent the peak values of their statistics. These values may characterize' the typical properties of the gas which is not directly affected by star formation. The median and mean line widths are $1.3 \mathrm{~km} \mathrm{~s}^{-1}$ and $1.5 \mathrm{~km} \mathrm{~s}^{-1}$, respectively (see Sect.4.2) while median and mean kinetic temperatures are $23 \mathrm{~K}$ and $26 \mathrm{~K}$, respectively (see Sect.4.3). Adopting the median temperature and line width, the corresponding spacings for thermal and turbulent cylinder fragmentation are $1.1 \mathrm{pc}$ and $2.18 \mathrm{pc}$. Adopting the means of temperature and line width, the corresponding spacings for thermal and turbulent cylinder fragmentation are $1.17 \mathrm{pc}$ and $2.5 \mathrm{pc}$. Therefore, for the median case, inclinations of $47^{\circ}$ and $32^{\circ}$ should be assumed to explain the observed spacings. For the mean case, inclinations of $51^{\circ}$ and $38^{\circ}$ are needed. Neither for the median nor the mean case, can the calculated thermal and turbulent spacings be explained by a consistent inclination.

The (quasi-) periodically spaced fragmentation in the ISF has been noticed by many previous studies from several tens of parsecs to a few percent of a parsec (e.g., Dutrey et al. 1991;
Johnstone \& Bally 1999; Takahashi et al. 2013). Dutrey et al. (1991) first presented an obvious periodical density structure with $\mathrm{C}^{18} \mathrm{O}$ and suggested the periodicity is a result of externally triggered and magnetically mediated cloud fragmentation. More recently, Takahashi et al. (2013) summarized the fragmentation in the entire Orion complex from large to small scale, including a spacing of $\sim 4.7^{\circ}$ (34 pc) corresponding to the separation between the Orion A and B giant molecular clouds (e.g., Maddalena et al. 1986), a spacing of $9^{\prime}-10^{\prime}(1.0-1.2 \mathrm{pc})$ corresponding to the separation of the large scale clumps, for example, OMC-1, OMC-2 (e.g., Dutrey et al. 1991; Hanawa et al. 1993), a spacing of about $2.5^{\prime}(0.3 \mathrm{pc})$ corresponding to the separation between small scale clumps (e.g., Johnstone \& Bally 1999), and the spacing corresponding to the dense cores, for example, $17^{\prime \prime}(0.034 \mathrm{pc})$ in OMC-2/3 and 30" (0.06 pc) in OMC-1 (e.g., Wiseman \& Ho 1998; Takahashi et al. 2013; Teixeira et al. 2016). They argued that fragmentation spacings on scales of $0.1-1 \mathrm{pc}$ are roughly consistent with thermal fragmentation, while on small scales $(<0.1 \mathrm{pc})$ fragmentation spacings are below those of the thermal fragmentation. Our data showing two levels, that is, $1.36 \pm 0.16 \mathrm{pc}$ and $0.86 \pm 0.14 \mathrm{pc}$, are consistent with turbulent and thermal cylinder fragmentation.

Because our $\mathrm{NH}_{3}$ observations could well resolve the spacings between the clumps/sub-clumps, the spacings identified by our $\mathrm{NH}_{3}$ observations are reliable. Lower-resolution observations may more clearly present the larger-scale fragmentation (i.e., Fig. 3 in Teixeira et al. 2016). Meanwhile, according to a high-fidelity map of G11.11-0.12, Kainulainen et al. (2013) suggested that on scales larger than $0.5 \mathrm{pc}$, fragmentation may still be affected by global instabilities which is in agreement with the model of a self-gravitating cylinder. Therefore, the two-level hierarchical cylinder fragmentation at scales of 0.86 and $1.36 \mathrm{pc}$ should be reasonable. Furthermore, this two-level fragmentation indicates that the clump separation is due to turbulent pressure confinement and the sub-clump separation is due to thermal pressure confinement; that is, turbulence should be dissipated from the scale of clumps to sub-clumps in the ISF. We studied the line width at different scales in a quiescent clump, OMC-2, in Appendix C. As the linear scale length decreases, the velocity dispersion decreases as is also deduced by many previous studies (i.e., Goodman et al. 1998; Barranco \& Goodman 1998).

\subsection{Kinematics from $\mathrm{NH}_{3}$}

Figure 3 presents the kinematics of the ISF derived from $\mathrm{NH}_{3}(1$, $1)$. The left panel displays velocity (color image) and the right panel indicates the intrinsic line width (i.e., for an individual hyperfine component, gray image). They are all derived by the $\mathrm{NH}_{3}$ $(1,1)$ fitting method in GILDAS. The two images are overlaid with $\mathrm{NH}_{3}(1,1)$ integrated intensity contours as in Fig. 1. Firstly, we can see that a clear velocity gradient runs along the main part of the ISF. Following the procedure outlined in Goodman et al. (1993), we fitted the velocity gradient as a simple linear form: $V_{\mathrm{LSR}}=v_{0}+a \times \delta \alpha+b \times \delta \beta$, where $\delta \alpha$ and $\delta \beta$ are offsets in right ascension and declination (in radians), and $v_{0}$ presents the systemic velocity of the cloud. The velocity gradient is therefore given by $\nabla v=\left(a^{2}+b^{2}\right)^{0.5} / D$ at a distance of $D$. The derived gradient is $0.6 \pm 0.08 \mathrm{~km} \mathrm{~s}^{-1} \mathrm{pc}^{-1}$ at $414 \mathrm{pc}$ using all the spectra with peak line flux larger than $5 \sigma$. This linear velocity fitting is based on a solid body approximation for the ISF. To check this assumption, we study the velocity residuals $\left(V_{\text {obs }}-V_{\text {rigid }}\right)$ in Appendix D. The velocity residuals do not present major departures from a linear velocity distribution. The observed velocity gradient is comparable to the overall rotation of the Orion A Molecular Cloud (e.g., 

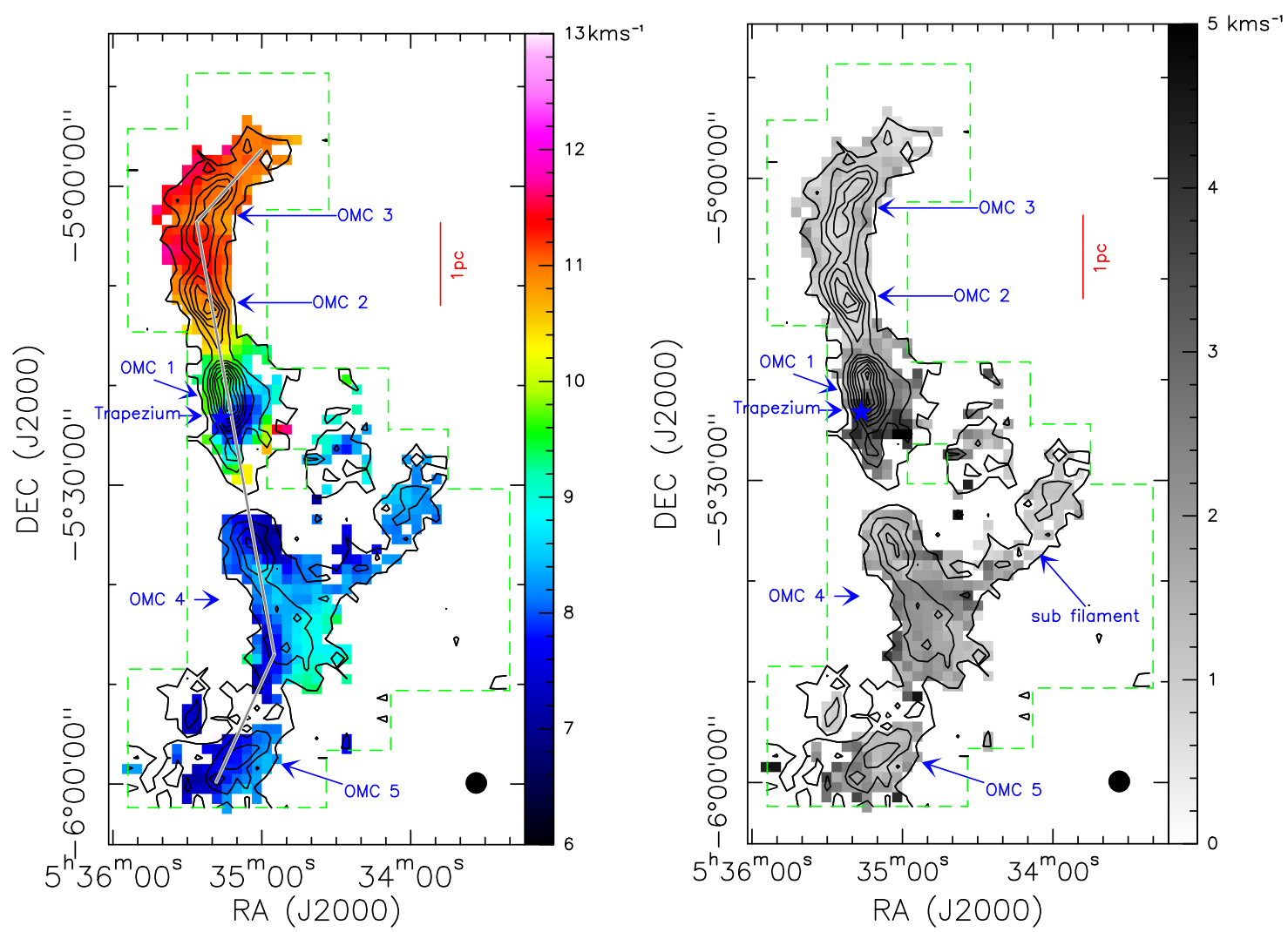

Fig. 3. Kinematics of the ISF derived from $\mathrm{NH}_{3}(1,1)$. Left panel: velocity map (color image) of the ISF. Right panel: FWHM line width map (gray image) of the ISF. Contours in each panel are the same as in the left panel of Fig. 1. The gray solid polyline in the left panel indicates the loci for the position-velocity diagram presented in Fig. 5.

Wilson et al. 2005). Wilson et al. (2005) suggested an increase in the inclination of the filament as a function of time with respect to the line of sight (see Sect.4.1). Taking the ISF as a homogeneous rigidly rotating cylinder, the ratio of rotational (around its center near $\delta \sim-5^{\circ} 30^{\prime}$ ) to gravitational energy is

$\beta=\frac{E_{\mathrm{rot}}}{E_{\mathrm{grav}}}=\frac{1}{2} \frac{M L^{2}}{12}\left(1+\frac{3}{2 x^{2}}\right) \omega^{2} / \frac{3}{2} \frac{G M^{2}}{L} f(x) \approx \frac{\omega^{2}}{4 \pi G \rho} \frac{L^{2}}{9 R^{2}}$,

where $G$ is the gravitational constant and $\rho, L, R$, and $\omega$ are the density, height, radius and the angular velocity of the cylinder. $x \equiv L / D, f(x) \sim 1$ (Bonnell et al. 1992).

We can also write this as

$\beta=\frac{3.0 \times 10^{-3} \omega_{-14}^{2}}{n_{4}} \frac{L^{2}}{9 R^{2}}$,

where, $\omega_{-14}$ is the angular velocity in units of $10^{-14} \mathrm{~s}^{-1}$ and $n_{4}$ is the gas density in units of $10^{4} \mathrm{~cm}^{-3}$ (Menten et al. 1984; Levshakov et al. 2013). Adopting an angular velocity of $1.94 \times 10^{-14} \mathrm{~s}^{-1}$ and an average density of the ISF of $10^{4} \mathrm{~cm}^{-3}$ (Bally et al. 1987; Dutrey et al. 1991; Berné et al. 2014), then $\beta$ $\sim 0.25$. Thus, the rotational energy is a small fraction of the gravitational energy.

From the line width image, three patterns are apparent. Firstly, the most dispersed region is located around the Trapezium in OMC-1. Secondly, in the northern part of the ISF, very low and also uniform intrinsic (for individual hyperfine components) dispersions $\left(\sigma \sim 0.4 \pm 0.08 \mathrm{~km} \mathrm{~s}^{-1}\right)$ are present. Very narrow line widths of the $\mathrm{NH}_{3}(1,1)$ main line are mostly found in

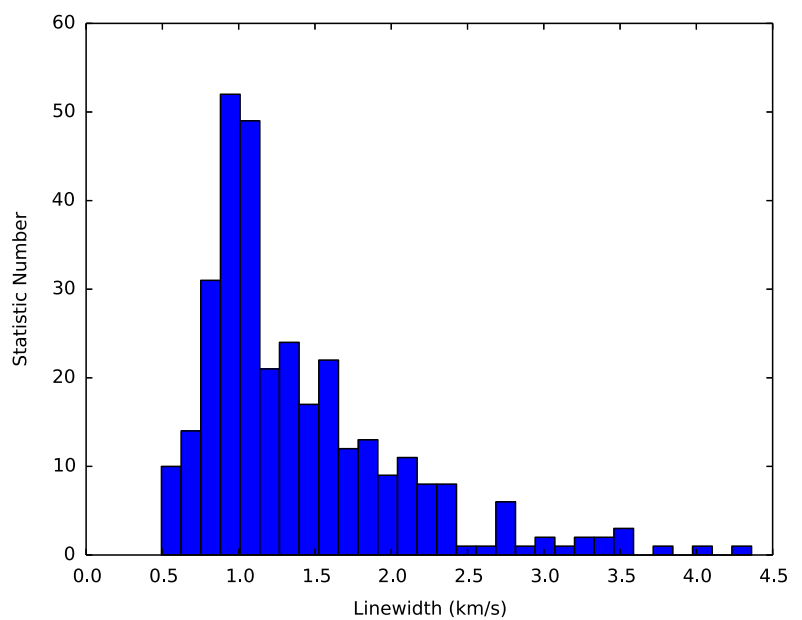

Fig. 4. Histograms of the intrinsic FWHM line widths of the $\mathrm{NH}_{3}(1,1)$ main lines. These line widths referring to individual hyperfine components are derived from the GILDAS built-in " $\mathrm{NH}_{3}(1,1)$ " fitting which can fit all 18 hyperfine components simultaneously.

this region. Thirdly, irregular and larger dispersion is present in the southern part of the ISF. This may mainly be because in this region $\mathrm{S} / \mathrm{Ns}$ are not as high. We study the FWHM line widths of the $\mathrm{NH}_{3}(1,1)$ main lines with a peak line flux threshold of $5 \sigma$ as summarized in Fig. 4. The line width refers to individual hyperfine component and is fitted using the GILDAS built-in " $\mathrm{NH}_{3}$ $(1,1)$ " fitting method. Obviously the line width distribution of the $\mathrm{NH}_{3}(1,1)$ gas has an outstanding peak around $1 \mathrm{~km} \mathrm{~s}^{-1}$. Therefore we adopted this line width as the typical width for the 


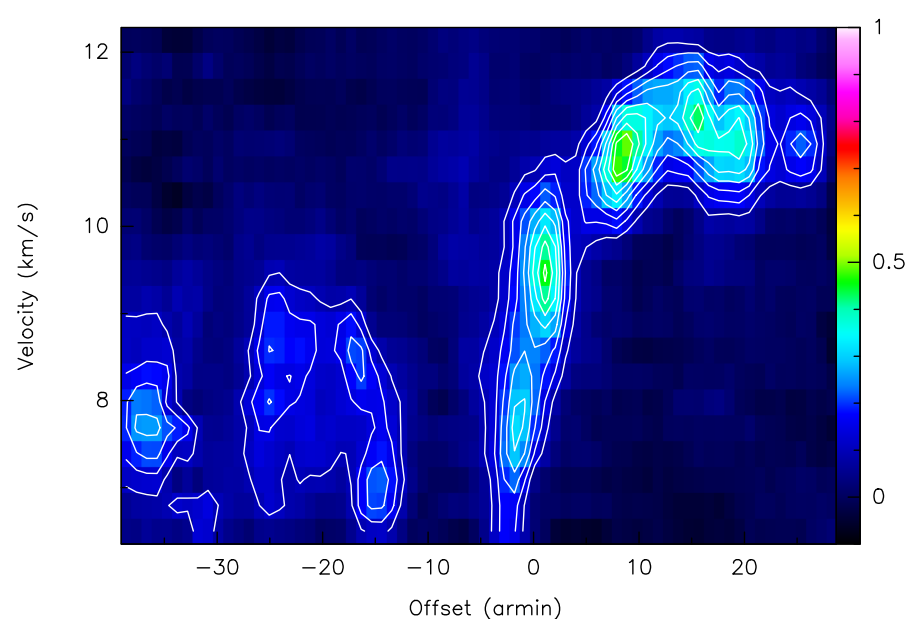

Fig. 5. Position-velocity (PV) diagram of the $\mathrm{NH}_{3}(1,1)$ main line along the gray solid poly-line in the left panel of Fig. 3. The cut runs through the ISF with negative offsets referring to the southern part of the filament. Offset 0 refers to the location of the Trapezium cluster. The threshold and step of the contours of the position-velocity diagram are all $10 \%$ of the peak flux.

fragmentation analysis in Sect. 4.1. The median and mean intrinsic line widths are $1.3 \mathrm{~km} \mathrm{~s}^{-1}$ and $1.5 \mathrm{~km} \mathrm{~s}^{-1}$, respectively.

To clearly show the kinematics along the ISF, we created the poly-line position-velocity (PV) diagram presented in Fig. 5. The loci of the PV diagram, which runs through the ridge of the ISF and crosses the cores, are illustrated by the gray solid lines in the left panel of Fig. 3. We averaged 5 pixels $\left(5^{\prime}\right)$ perpendicular to the loci in order to present the integral kinematics and to make sure that the exact location of the poly-line, chosen by eye, is not a critical parameter. From the diagram, we can see that, (1) there is an overall velocity gradient along the ISF as shown in the velocity image (left panel of Fig. 3); (2) bounded by OMC1 , the features of the northern part are coherent and compact, and the southern ones are diffuse and faint; and (3) the two subclumps in the OMC-1 show a large difference in velocity.

Filamentary structures of MCs may be seen as accretion channels to their associated clumps and lead to a seesaw velocity distribution (e.g., Kirk et al. 2013; Zhang et al. 2015). Therefore, to further examine the gas kinematics, Fig. 6 presents the centroid velocity obtained by the main lines of $\mathrm{NH}_{3}(1,1)$. We firstly excluded the spectra with peak line flux less than $5 \sigma$ to avoid spurious results. We then averaged the spectra within a $1^{\prime}$ wide belt in declination along right ascension which is roughly perpendicular to the main filamentary structure. Finally, single Gaussian fits were used to get the centroid velocities of these averaged spectra. The result is summarized in Fig. 6 with blue dots and lines. Beside the overall motion (velocity gradient), fluctuations are also seen along the ISF. The locations of OMC-1 to 5 are labeled in this figure and are all found near the local extrema of the fluctuations. This may hint at gas flows toward the clumps in the ISF but may also arise from effects related to star formation. Besides, we also derive the centroid velocities of IRAM ${ }^{13} \mathrm{CO}$ (2-1) data with the same method as for $\mathrm{NH}_{3}$ and summarize this in Fig. 6 with red dots and lines. The large velocity shifts and fluctuations are roughly the same as for $\mathrm{NH}_{3}$. However, there is a striking velocity jump $\left(\sim 2 \mathrm{~km} \mathrm{~s}^{-1}\right)$ of $\mathrm{NH}_{3}$ relative to ${ }^{13} \mathrm{CO}$ around $\mathrm{OMC}-1$. According to ${ }^{13} \mathrm{CO}$ observations in Orion A (e.g., Bally et al. 1987; Berné et al. 2014), the northern part of the Orion A molecular cloud is a coherent structure. It is not likely that ${ }^{13} \mathrm{CO}$ is from a separate group of clouds along

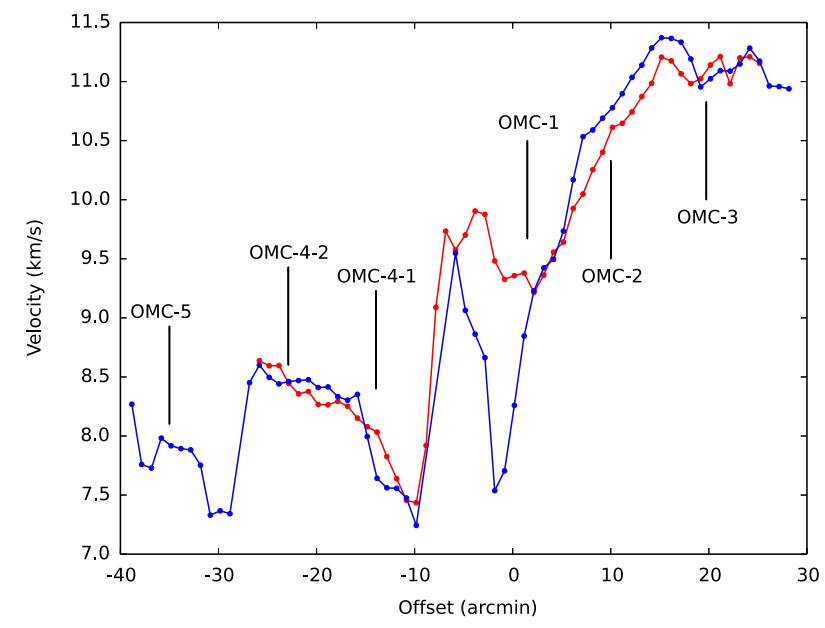

Fig. 6. The centroid velocities of the $\mathrm{NH}_{3}(1,1)$ (blue dots and lines) and ${ }^{13} \mathrm{CO}(1-0)$ (red dots and lines) averaged in $1^{\prime}$-width belts in declination along the ISF. Offset 0 refers to the location of the Trapezium cluster. The locations of OMC-1 to 5 are also displayed in this panel.

the line of sight. Considering the strong $\mathrm{NH}_{3}$ emission originating from OMC-1's dense hot core with a radial velocity of $\sim 5 \mathrm{~km} \mathrm{~s}^{-1}$, while that of the nearby Orion ridge, which dominates the ${ }^{13} \mathrm{CO}$ emission, is near $8 \mathrm{~km} \mathrm{~s}^{-1}$ (e.g., Genzel et al. 1982; Wiseman \& Ho 1998), the discrepancy is readily explained.

\subsection{Temperature}

The lack of allowed radiative transitions between the $K=1$ and 2 ladders of $\mathrm{NH}_{3}$ makes their relative populations highly sensitive to collisions, therefore making them a good estimator of the gas kinetic temperature. The rotation temperature of the two levels is derived following the method in Ho \& Townes (1983). The integrated optical depth of the main feature of the $\mathrm{NH}_{3}(1,1)$ transition, excluding the satellite lines, is derived by the GILDAS built-in " $\mathrm{NH}_{3}(1,1)$ " fitting method. Subsequently, we used the formula below to obtain the rotational temperature,

$$
\begin{aligned}
& T_{\text {rot }}(2,2: 1,1)= \\
& -41.5 \div \ln \left[\frac{-0.282}{\tau_{\mathrm{m}}(1,1)} \ln \left[1-\frac{\Delta T_{\mathrm{a}}(2,2, m)}{\Delta T_{\mathrm{a}}(1,1, m)}\right]\left(1-\exp ^{-\tau_{\mathrm{m}}(1,1)}\right)\right],
\end{aligned}
$$

where $\Delta T$ is the main line intensity and $\tau_{\mathrm{m}}$ denotes the main group opacity derived using the GILDAS built-in ' $\mathrm{NH}_{3}(1,1)$ ' fitting. $\triangle T$ of $\mathrm{NH}_{3}(1,1)$ and $\mathrm{NH}_{3}(2,2)$ are derived using the GILDAS built-in 'GAUSS' fitting. The mean uncertainty of the derived temperature is $2 \mathrm{~K}$.

The color image in the left panel of Fig. 7 presents the rotational temperature $\left(T_{\mathrm{R}}\right)$ distribution of the ISF with overlaid $\mathrm{NH}_{3}$ $(1,1)$ integrated intensity contours as in Fig. 1. $T_{\mathrm{R}}$ exceeds $40 \mathrm{~K}$ towards several positions. There the intensities of $\mathrm{NH}_{3}(2,2)$ are even stronger than those of $\mathrm{NH}_{3}(1,1)$ and they mainly come from OMC-1. As an example, nine spectra around the Trapezium are presented in the right-bottom panel of Fig. 7 (black spectra for $\mathrm{NH}_{3}(1,1)$, red spectra for $\left.\mathrm{NH}_{3}(2,2)\right)$. From the left panel we can see, the gas around the Trapezium cluster is characterized by $T_{\mathrm{R}} \sim 30-40 \mathrm{~K}$. The gas in OMC-2, OMC-3, and the northern part of OMC-4 has a lower $T_{\mathrm{R}}$ of about $20 \mathrm{~K}$ or even lower than that. In OMC-2 to 4, we can see higher $T_{\mathrm{R}}$ at the boundaries and lower $T_{\mathrm{R}}$ in the interior of these clumps. This may illustrate that 

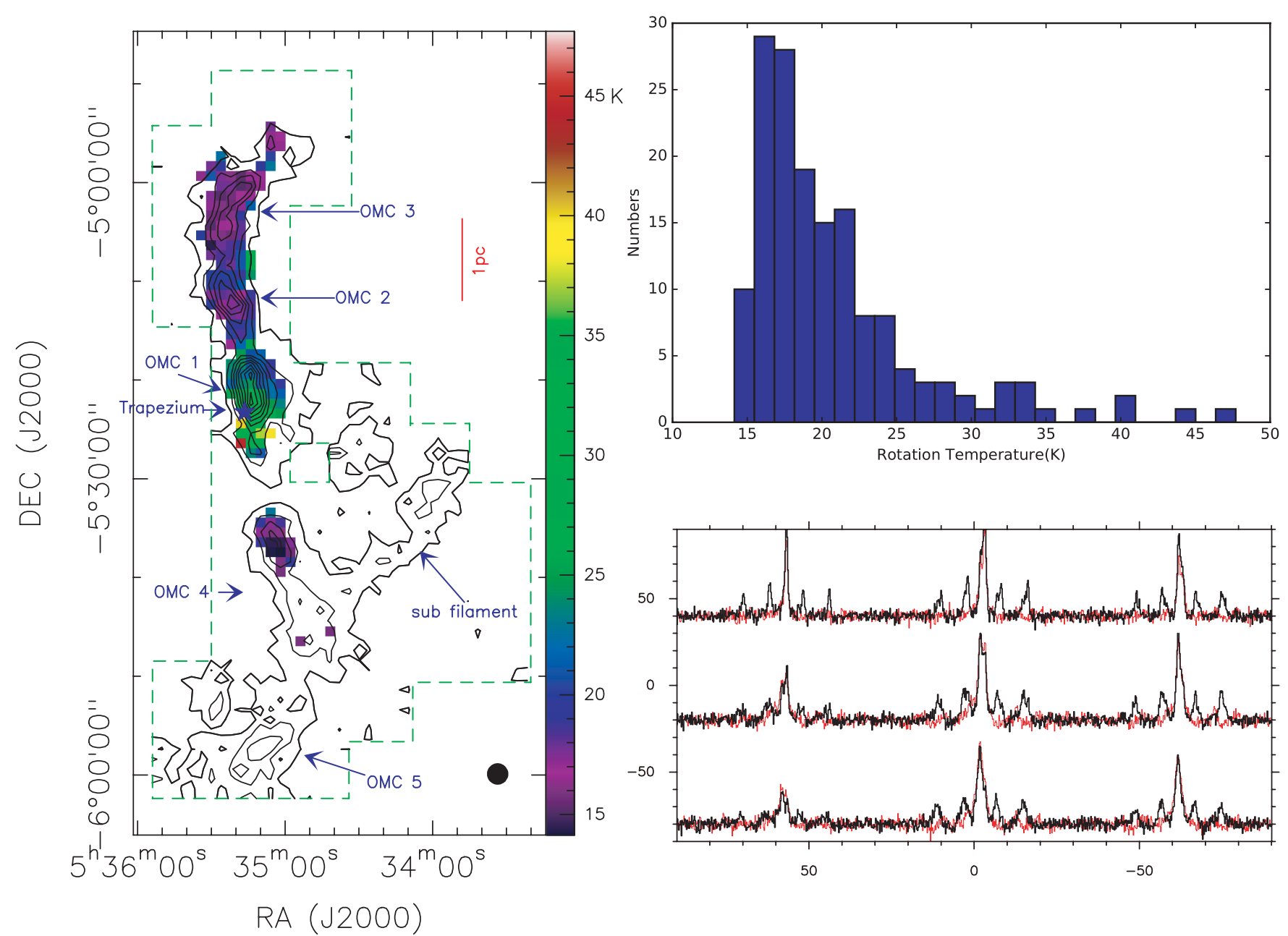

Fig. 7. Left panel: temperature distribution along the ISF overlaid with integrated intensity contours as in the left panel of Fig. 1. Most of the temperatures are about or below $20 \mathrm{~K}$. Temperatures exceed $40 \mathrm{~K}$ at several positions. There the $\mathrm{NH}_{3}(2,2)$ lines are even stronger than those of $\mathrm{NH}_{3}(1,1)$ (see the lower right panel). Top right panel: histogram of the rotation temperatures derived from $\mathrm{NH}_{3}$. Lower right panel: the $\mathrm{NH}_{3}$ $(1,1)$ (black profiles) and $\mathrm{NH}_{3}(2,2)$ (red profiles) spectra around the Trapezium. The reference position corresponds to RA: 05:35:14, Dec: $-05: 22: 24(\mathrm{~J} 2000)$, the spacing is one arcminute.

heating originates mainly from the outside and that these clumps may still be in an early evolutionary stage.

A statistical distribution of $T_{\mathrm{R}}$ is summarized in the topright panel of Fig. 7 and shows a typical value of about $16 \mathrm{~K}$, corresponding to a kinetic temperature of $18 \mathrm{~K}$ (following Tafalla et al. 2004), which has been used in Sect. 4.1 to calculate expected clump separations inside a cloud of cylindric morphology. The median and mean values are $19 \mathrm{~K}$ and $20.8 \mathrm{~K}$, corresponding to a kinetic temperature of $23 \mathrm{~K}$ and $26 \mathrm{~K}$ (following Tafalla et al. 2004).

\subsection{A brief comparison with GBT data}

While this work was in progress, Friesen et al. (2017) published ammonia data of the ISF obtained with the Green Bank Telescope. This map, taken with a resolution of $32^{\prime \prime}$, extends slightly further to the south but is limited to the narrow ridge and does not include the western protrusion of the filament we see between $\delta \sim-5^{\circ} 25^{\prime}$ and $\delta \sim-5^{\circ} 40^{\prime}$. Agreement in the overall velocity, line width, and kinetic temperature distributions is fairly good. We also identify the cloud displaced $5^{\prime}-10^{\prime}$ eastwards of the backbone of the filament near $\delta \sim-5^{\circ} 52^{\prime}$.

\section{Summary}

We have mapped the ISF in $\mathrm{NH}_{3}(1,1)$ and $(2,2)$ located at the northern end of the Orion A molecular cloud. The uniformly and fully-sampled observations cover a region of about 1.2 degrees in declination and about 0.6 degrees in right ascension. The main conclusions of this work are as follows.

The $\mathrm{NH}_{3}$ morphology of the ISF is associated with the Herschel infrared dust ridge and also shows an ISF morphology. A 2-pc-length "sub-filament" extruding toward the northwest of OMC-4 also shows a clear corresponding far infrared feature and is detected for the first time in $\mathrm{NH}_{3}$.

We identify 6 clumps and 11 sub-clumps in the integrated intensity maps of $\mathrm{NH}_{3}(1,1)$. We find the clumps and sub-clumps are all separated not randomly but in roughly equal intervals. Comparing our data with (quasi-) periodically spaced fragmentation in the ISF revealed by previous studies, we propose that hierarchical fragmentation around the clump scale $(\sim 1 \mathrm{pc})$ shows two levels, that is, clumps at separations of $1.36 \pm 0.16 \mathrm{pc}$ and sub-clumps with a spacing of $0.86 \pm 0.14 \mathrm{pc}$. Adopting an inclination of $60^{\circ}$, this two-level fragmentation is consistent with turbulent and thermal fragmentation inside a gas cloud with cylindric morphology. 
A velocity gradient of $0.6 \mathrm{~km} \mathrm{~s}^{-1} \mathrm{pc}^{-1}$ runs along the ISF, likely due to an overall rotation of the Orion A cloud. Using this velocity, we compared rotational and gravitational energy of the ISF. The ratio is about 0.25 , demonstrating that the rotational energy is a small fraction of the gravitational energy. We made an averaged centroid velocity diagram along the ISF to check the roles of filaments as the accretion channels to clumps. From the diagram, fluctuations are seen along the ISF. OMC-1 to 5 are all located near local extrema of the fluctuations. This may indicate gas flows toward the clumps in the ISF but may also be explained in terms of star forming activity. Moreover, a striking velocity jump of $\mathrm{NH}_{3}$ relative to ${ }^{13} \mathrm{CO}$ around the $\mathrm{OMC}-1$ is found in this diagram. The explanation is that $\mathrm{NH}_{3}$ is enhanced by hot dense clumps while ${ }^{13} \mathrm{CO}$ is mainly associated with less dense cooler gas.

The derived $\mathrm{NH}_{3}(1,1)$ and $(2,2)$ rotation temperatures in the OMC-1 are about 30-40 K. Lower rotation temperatures (below $20 \mathrm{~K}$ ) are present at the northern and the southern parts of the ISF. OMC-2, OMC-3, and northern part of OMC-4 show larger temperatures at the boundaries and lower temperatures in the interior of these clumps. This may illustrate that heating mainly occurs from the outside and that these clumps may still reside in an early evolutionary stage.

Acknowledgements. This work was funded by the Program of the Light in China's Western Region (LCRW) under grant Nos. and 2015-XBQN-B-03 and the National Nature Science foundation of China under grant 11433008 , 11603063 , partly supported by the National Natural Science foundation of China under grant 11373062, 11303081. K.Q. acknowledges the support from National Natural Science Foundation of China (NSFC) through grants NSFC 11473011 and NSFC 11590781

\section{References}

André, P., Men'shchikov, A., Bontemps, S., et al. 2010, A\&A, 518, L102 André, P., Di Francesco, J., Ward-Thompson, D., et al. 2014, Protostars and Planets VI, 27

Bally, J. 2008, in Overview of the Orion Complex, ed. B. Reipurth, 459 Bally, J., Langer, W. D., Stark, A. A., \& Wilson, R. W. 1987, ApJ, 312, L45

Barranco, J. A., \& Goodman, A. A. 1998, ApJ, 504, 207

Berné, O., Marcelino, N., \& Cernicharo, J. 2014, ApJ, 795, 13

Bonnell, I., Arcoragi, J.-P., Martel, H., \& Bastien, P. 1992, ApJ, 400, 579

Cesaroni, R., \& Wilson, T. L. 1994, A\&A, 281, 209

Chandrasekhar, S., \& Fermi, E. 1953, ApJ, 118, 116

Danby, G., Flower, D. R., Valiron, P., Schilke, P., \& Walmsley, C. M. 1988 MNRAS, 235, 229
Dutrey, A., Duvert, G., Castets, A., et al. 1991, A\&A, 247, L9

Evans, II, N. J. 1999, ARA\&A, 37, 311

Friesen, R. K., Pineda, J. E., co-PIs, et al. 2017, ApJ, 843, 63

Genzel, R., \& Stutzki, J. 1989, ARA\&A, 27, 41

Genzel, R., Ho, P. T. P., Bieging, J., \& Downes, D. 1982, ApJ, 259, L103

Goodman, A. A., Benson, P. J., Fuller, G. A., \& Myers, P. C. 1993, ApJ, 406 528

Goodman, A. A., Barranco, J. A., Wilner, D. J., \& Heyer, M. H. 1998, ApJ, 504, 223

Hanawa, T., Nakamura, F., Matsumoto, T., et al. 1993, ApJ, 404, L83

Ho, P. T. P., \& Townes, C. H. 1983, ARA\&A, 21, 239

Hunter, J. D. 2007, Comput. Sci. Eng., 9, 90

Inutsuka, S.-I., \& Miyama, S. M. 1992, ApJ, 388, 392

Jackson, J. M., Finn, S. C., Chambers, E. T., Rathborne, J. M., \& Simon, R. 2010, ApJ, 719, L185

Johnstone, D., \& Bally, J. 1999, ApJ, 510, L49

Johnstone, D., \& Bally, J. 2006, ApJ, 653, 383

Kainulainen, J., Ragan, S. E., Henning, T., \& Stutz, A. 2013, A\&A, 557, A120

Kirk, H., Myers, P. C., Bourke, T. L., et al. 2013, ApJ, 766, 115

Kounkel, M., Hartmann, L., Loinard, L., et al. 2017, ApJ, 834, 142

Levshakov, S. A., Henkel, C., Reimers, D., et al. 2013, A\&A, 553, A58

Li, D. L., Esimbek, J., Zhou, J. J., et al. 2014, A\&A, 567, A10

Lu, X., Zhang, Q., Liu, H. B., Wang, J., \& Gu, Q. 2014, ApJ, 790, 84

Maddalena, R. J., Morris, M., Moscowitz, J., \& Thaddeus, P. 1986, ApJ, 303, 375

Menten, K. M., Walmsley, C. M., Kruegel, E., \& Ungerechts, H. 1984, A\&A, 137,108

Menten, K. M., Reid, M. J., Forbrich, J., \& Brunthaler, A. 2007, A\&A, 474, 515

Ohashi, S., Sanhueza, P., Chen, H.-R. V., et al. 2016, ApJ, 833, 209

Ostriker, J. 1964, ApJ, 140, 1056

Ott, S. 2010, in Astronomical Data Analysis Software and Systems XIX, eds.

Y. Mizumoto, K.-I. Morita, \& M. Ohishi, ASP Conf. Ser., 434, 139

Pineda, J. E., Rosolowsky, E. W., \& Goodman, A. A. 2009, ApJ, 699, L134

Schneider, S., \& Elmegreen, B. G. 1979, ApJS, 41, 87

Shirley, Y. L. 2015, PASP, 127, 299

Stutz, A. M., \& Kainulainen, J. 2015, A\&A, 577, L6

Tafalla, M., Myers, P. C., Caselli, P., \& Walmsley, C. M. 2004, A\&A, 416, 191

Takahashi, S., Ho, P. T. P., Teixeira, P. S., Zapata, L. A., \& Su, Y.-N. 2013, ApJ, 763, 57

Tan, J. C., Beltrán, M. T., Caselli, P., et al. 2014, Protostars and Planets VI, 149 Teixeira, P. S., Takahashi, S., Zapata, L. A., \& Ho, P. T. P. 2016, A\&A, 587, A47 Walmsley, C. M., \& Ungerechts, H. 1983, A\&A, 122, 164

Wang, K., Zhang, Q., Testi, L., et al. 2014, MNRAS, 439, 3275

Williams, J. P., de Geus, E. J., \& Blitz, L. 1994, ApJ, 428, 693

Wilson, B. A., Dame, T. M., Masheder, M. R. W., \& Thaddeus, P. 2005, A\&A, 430, 523

Wiseman, J. J., \& Ho, P. T. P. 1998, ApJ, 502, 676

Zhang, Q., Wang, Y., Pillai, T., \& Rathborne, J. 2009, ApJ, 696, 268

Zhang, Q., Wang, K., Lu, X., \& Jiménez-Serra, I. 2015, ApJ, 804, 141

Zinnecker, H., \& Yorke, H. W. 2007, ARA\&A, 45, 481 


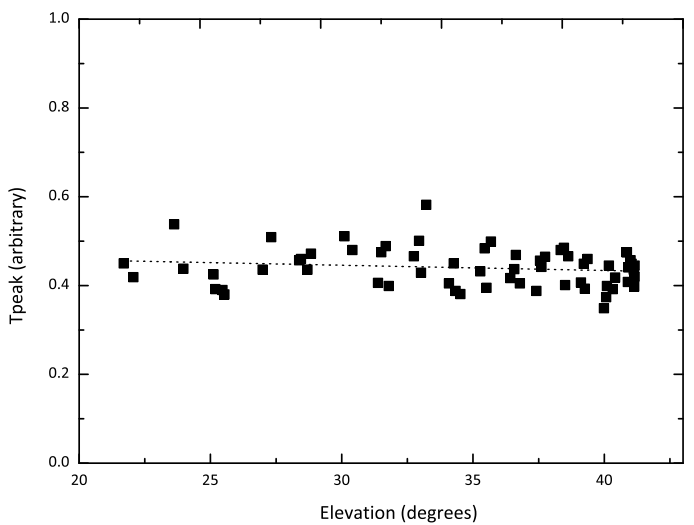

Fig. A.1. Uncorrected $\mathrm{NH}_{3}(1,1)$ main line intensities against elevation of repeated observations toward the reference position. The position corresponds to RA: 05:35:14, Dec: -05:22:24 (J2000). The statistical error of the flux is about $9.5 \%$. The dotted line indicates a linear fit.

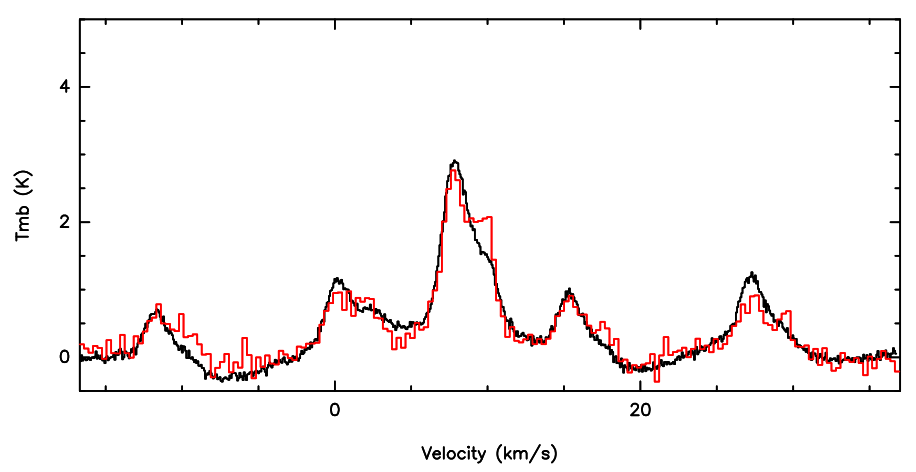

Fig. A.2. The $\mathrm{NH}_{3}(1,1)$ spectra from the GBT (black) and our data (red, multiplied by 5.31) toward the reference position RA: 05:35:14, Dec: -05:22:24 (J2000).

\section{Appendix A: Calibration stability}

The system temperature was calibrated against a signal injected by a noise diode, whose temperature is commonly determined by hot (ambient temperature) and cold (liquid nitrogen) loads. Because of a hardware problem, the output power of the diode noise source was abnormal but could maintain a constant value during the observations. To check the calibration stability, we observed the reference position (with $(0,0)$ offset) every $2-3 \mathrm{~h}$. The spectral line fluxes turned out to be stable but no absolute flux calibration could be obtained from the data themselves. All observations of the reference position were made in the OTF mode of a small area of $3^{\prime} \times 3^{\prime}$. We regridded the data and then fitted the $\mathrm{NH}_{3}(1,1)$ main lines (the central group of $\mathrm{NH}_{3}(1$, 1) hyperfine components) to present the peak intensity distribution against elevation (Fig. A.1). We can see in Fig. A.1 that there is no significant systematic variation and the dispersion is relatively small. The statistical error of the peak intensities is $9.5 \%$.

In order to calibrate our data to the main beam brightness temperature $\left(T_{\mathrm{mb}}\right)$ scale, we conducted a comparative analysis between the GBT (Friesen et al. 2017) and our $\mathrm{NH}_{3}(1,1)$ spectra. The GBT data were calibrated to $T_{\mathrm{mb}}$ scale with an estimated calibration uncertainty of about $10 \%$ (Friesen et al. 2017). We smoothed the GBT data to our beamsize using the "XY_MAP" routine in GILDAS. For the process our spectral cube was used as a template. We fitted the $\mathrm{NH}_{3}(1,1)$

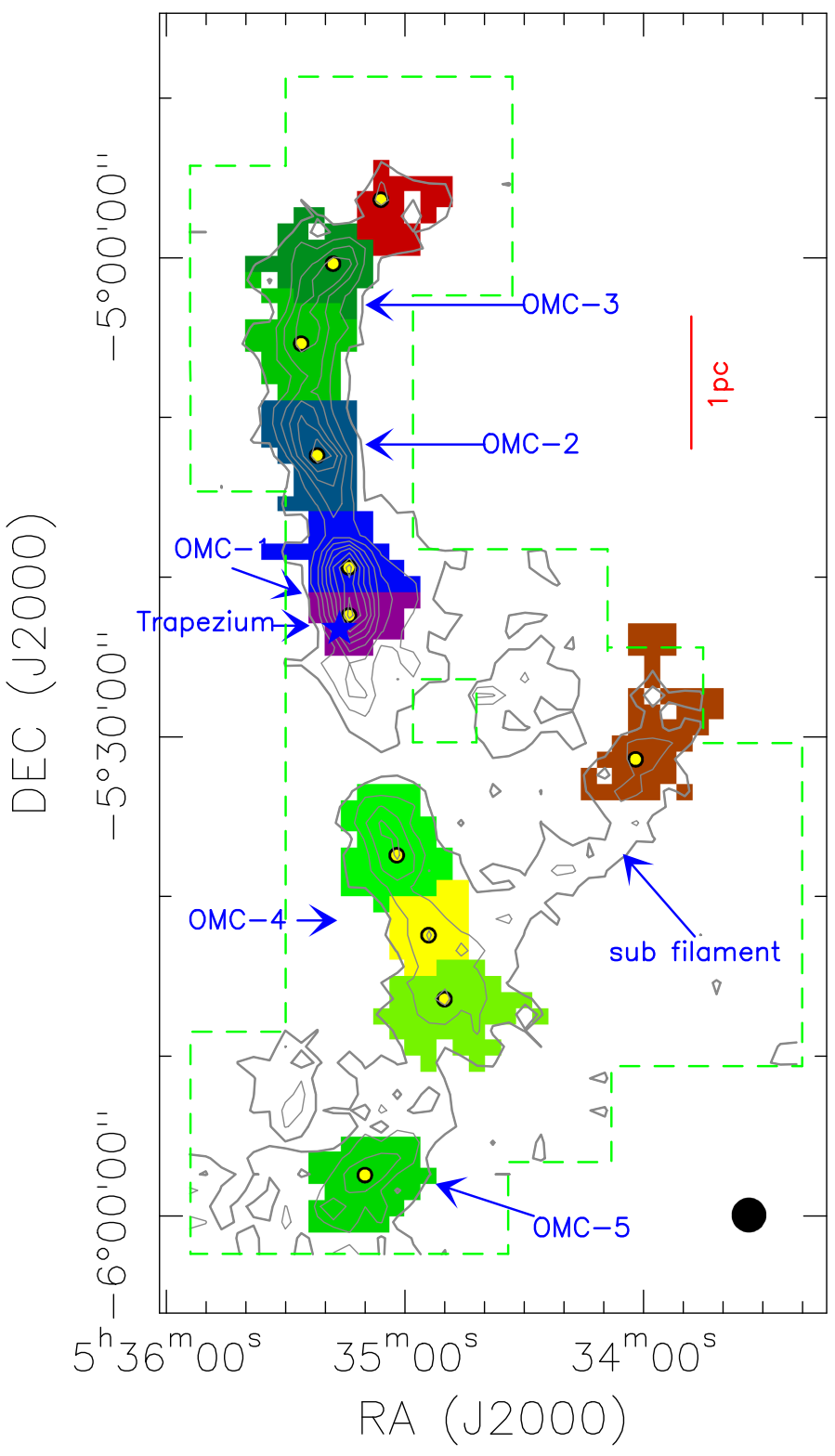

Fig. B.1. The colorized pixels illustrate the areas of the 11 substructures identified by the clumpfind algorithm. Contours are the same as in the left panel of Fig. 1. Yellow filled circles are same as in the right panel of Fig. 2.

main lines of the reference position and the derived peak line intensities are $2.46 \mathrm{~K}$ and $0.46 \mathrm{~K}$ for the GBT and our spectra respectively. Therefore our $\mathrm{NH}_{3}$ data were all multiplied by the factor of $2.46 / 0.46 \approx 5.31$. The GBT spectrum (black) and our spectrum (red, multiplied by 5.31) of the reference position are illustrated in Fig. A.2. We estimate our calibration uncertainty to be $\sqrt{(9.5 \%)^{2}+(10 \%)^{2}} \approx 14 \%$.

\section{Appendix B: The sub-structures identified by the clumpfind algorithm}

To more clearly present the sub-structures identified by the clumpfind algorithm, we colorized the pixels used to define the sub-structures in Fig. B.1. We can see these sub-structures are all related to the emission peaks and are composed of a considerable number of pixels. We should note that there are three substructures identified in OMC-4. The separation of the lower two 

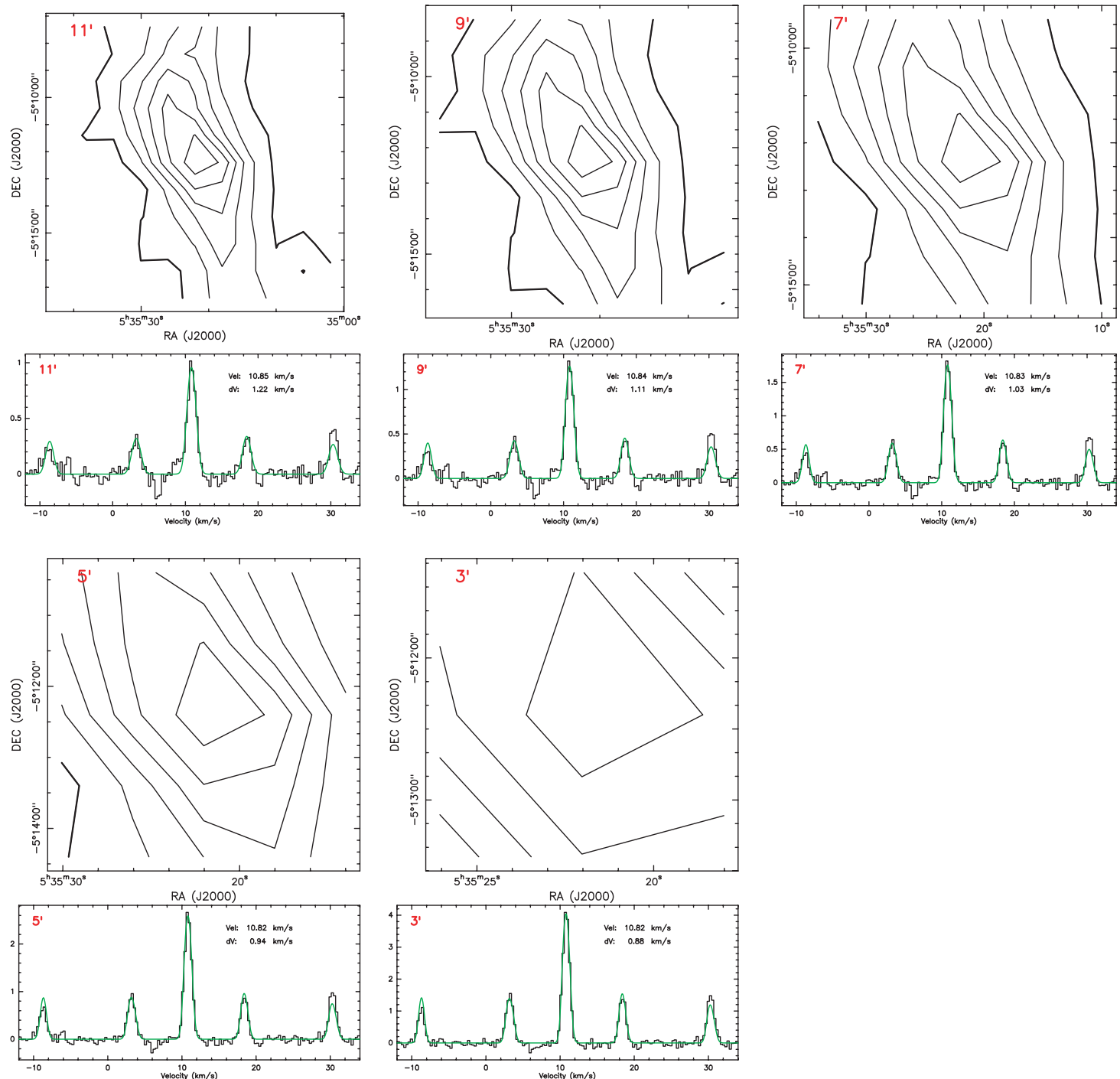

Fig. C.1. From left to right and top to bottom, the contours are the integrated intensity (zeroth moment) maps of $\mathrm{NH}_{3}(1,1)$ with side lengths of $11^{\prime}, 9^{\prime}, 7^{\prime}, 5^{\prime}$, and $3^{\prime}$ respectively. These images are all centered at the red cross in OMC-2 illustrated in the right panel of Fig. 2 . The integration range of each panel is $6.5<V L S R<12.5 \mathrm{~km} \mathrm{~s}^{-1}$. Contours start at $0.96 \mathrm{~K} \mathrm{~km} \mathrm{~s}^{-1}(4 \sigma)$ and go up in steps of $0.96 \mathrm{~K} \mathrm{~km} \mathrm{~s}^{-1}$. The spectra (black) overlaid with their fitting curves (green) are the averaged spectra within the regions corresponding to their upper panels.

sub-structures in OMC-4 may not be quite that obvious. There is indeed an emission peak at the center of OMC-4 beside the northern and southern ones.

\section{Appendix C: Dissipation of turbulent energy}

Since the clump OMC-2 exhibits lower temperature and simpler structure (OMC-2 has only one sub-clump) than the other clumps, we used this relatively quiescent clump to roughly estimate the velocity dispersion at different scales. We averaged the spectra within squared regions with side lengths of $11^{\prime}, 9^{\prime}, 7^{\prime}, 5^{\prime}$, and $3^{\prime}$ respectively which are all centered at the red cross in OMC-2 illustrated in the right panel of Fig. 2 (see the images in Fig. C.1). We fitted the averaged spectrum using the GILDAS built-in " $\mathrm{NH}_{3}(1,1)$ " fitting method (see the spectra in Fig. C.1). The derived intrinsic line widths referring to individual hyperfine components, $\Delta V_{\text {obs }}$, are $1.22 \pm 0.062 \mathrm{~km} \mathrm{~s}^{-1}, \quad 1.11 \pm 0.046 \mathrm{~km} \mathrm{~s}^{-1}, \quad 1.03 \pm 0.032 \mathrm{~km} \mathrm{~s}^{-1}$, $0.94 \pm 0.022 \mathrm{~km} \mathrm{~s}^{-1}$, and $0.88 \pm 0.017 \mathrm{~km} \mathrm{~s}^{-1}$ respectively. All the results above are summarized in Table C.1. The results are also displayed in Fig. C.2 in the form of $\sigma_{\mathrm{V}} / \sigma_{\mathrm{T}}$ versus side length, where, $\sigma_{\mathrm{T}}$ is the thermal velocity dispersion, which is commonly expressed in units of the the sound speed as $c_{\mathrm{s}}=$ $\sqrt{k T / \mu_{H} m_{H}}$. The mean temperature of OMC-2 derived from the averaged spectra of $\mathrm{NH}_{3}(1,1)$ and $(2,2)$ is about $20 \mathrm{~K}$. We can see that as the size length decreases, the velocity dispersion decreases and this decrease is approximately linear. Thus, the turbulence dissipates in the inner part of the ISF and we speculate that the thermal pressure of gas becomes more dominant.

We can see that in Fig. C.1, absorption features are present at $6 \mathrm{~km} \mathrm{~s}^{-1}$ and $20 \mathrm{~km} \mathrm{~s}^{-1}$. In fact, the right side of the main line and all satellite lines present potential absorption features. However, the main line with broader line width covers the absorption feature. By checking the raw data, these features are not from emission by the reference position. However, the S/Ns of these features are all lower than 1.8 and they are therefore not analyzed in this work. 


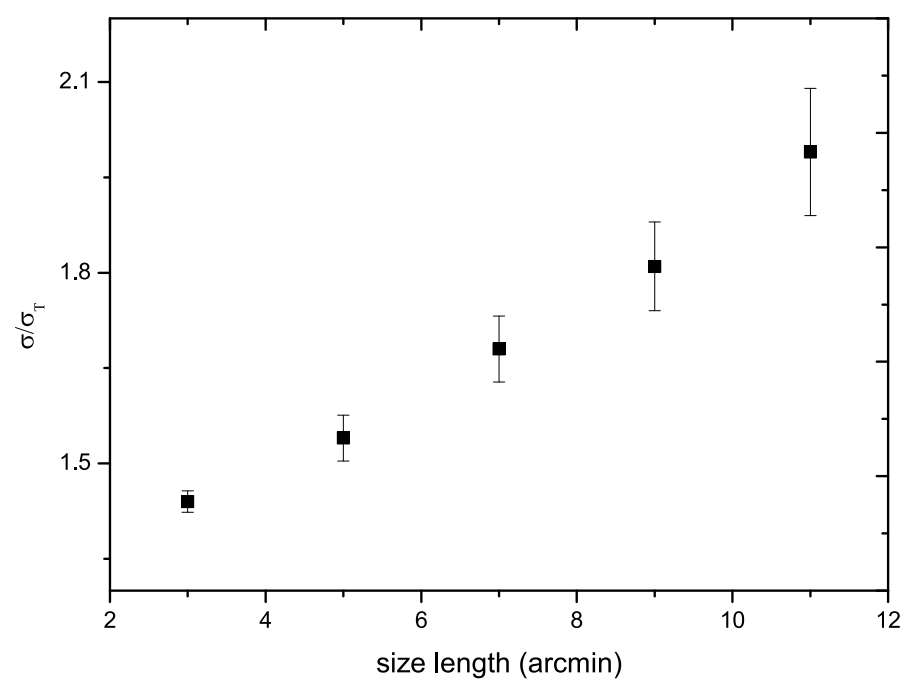

Fig. C.2. The ratio of observed to thermal velocity dispersion, $\sigma_{\mathrm{V}} / \sigma_{\mathrm{T}}$, derived from the averaged spectra in Fig. C.1 against the side length of the averaged regions.

Table C.1. Intrinsic (referring to individual hyperfine components) FWHM line widths, $\Delta V_{\text {obs }}$, associated velocity dispersions, $\sigma_{\mathrm{V}}$, and ratios between these velocity dispersions and the thermal velocity dispersion for the $\mathrm{NH}_{3}(1,1)$ data displayed in Fig. C.1.

\begin{tabular}{lccc}
\hline \hline $\operatorname{size}\left({ }^{\prime}\right)$ & $\Delta V_{\mathrm{obs}}\left(\mathrm{km} \mathrm{s}^{-1}\right)$ & $\sigma_{\mathrm{V}}\left(\mathrm{km} \mathrm{s}^{-1}\right)$ & $\sigma_{\mathrm{V}} / \sigma_{\mathrm{T}}$ \\
\hline 11 & $1.22 \pm 6.15 \mathrm{e}-2$ & $0.52 \pm 0.026$ & $1.99 \pm 0.10$ \\
9 & $1.11 \pm 4.55 \mathrm{e}-2$ & $0.47 \pm 0.019$ & $1.81 \pm 0.07$ \\
7 & $1.03 \pm 3.20 \mathrm{e}-2$ & $0.44 \pm 0.014$ & $1.68 \pm 0.05$ \\
5 & $0.94 \pm 2.22 \mathrm{e}-2$ & $0.40 \pm 0.009$ & $1.54 \pm 0.04$ \\
3 & $0.88 \pm 1.69 \mathrm{e}-2$ & $0.37 \pm 0.007$ & $1.44 \pm 0.03$ \\
\hline
\end{tabular}

\section{Appendix D: The residuals of the velocity fitting}

The linear velocity fitting is based on the assumption that the ISF is a rigid body. To check this assumption, we present the distribution of the velocity residuals between the observed velocity and the fitting velocity in Fig. D.1 and also the statistics of the velocity residuals in Fig. D.2. From Fig. D.1, we can see that most of the velocity residuals are distributed in the range $V_{\text {obs }}-V_{\text {rigid }}=-1 \mathrm{~km} \mathrm{~s}^{-1}$ to $1 \mathrm{~km} \mathrm{~s}^{-1}$ which is even more clearly presented in Fig. D.2. The larger velocity residuals are mainly negative and mostly located at the southern part of OMC-1 and the northern part of OMC-4. In general, the velocity residuals do not present major departures from a linear velocity distribution.

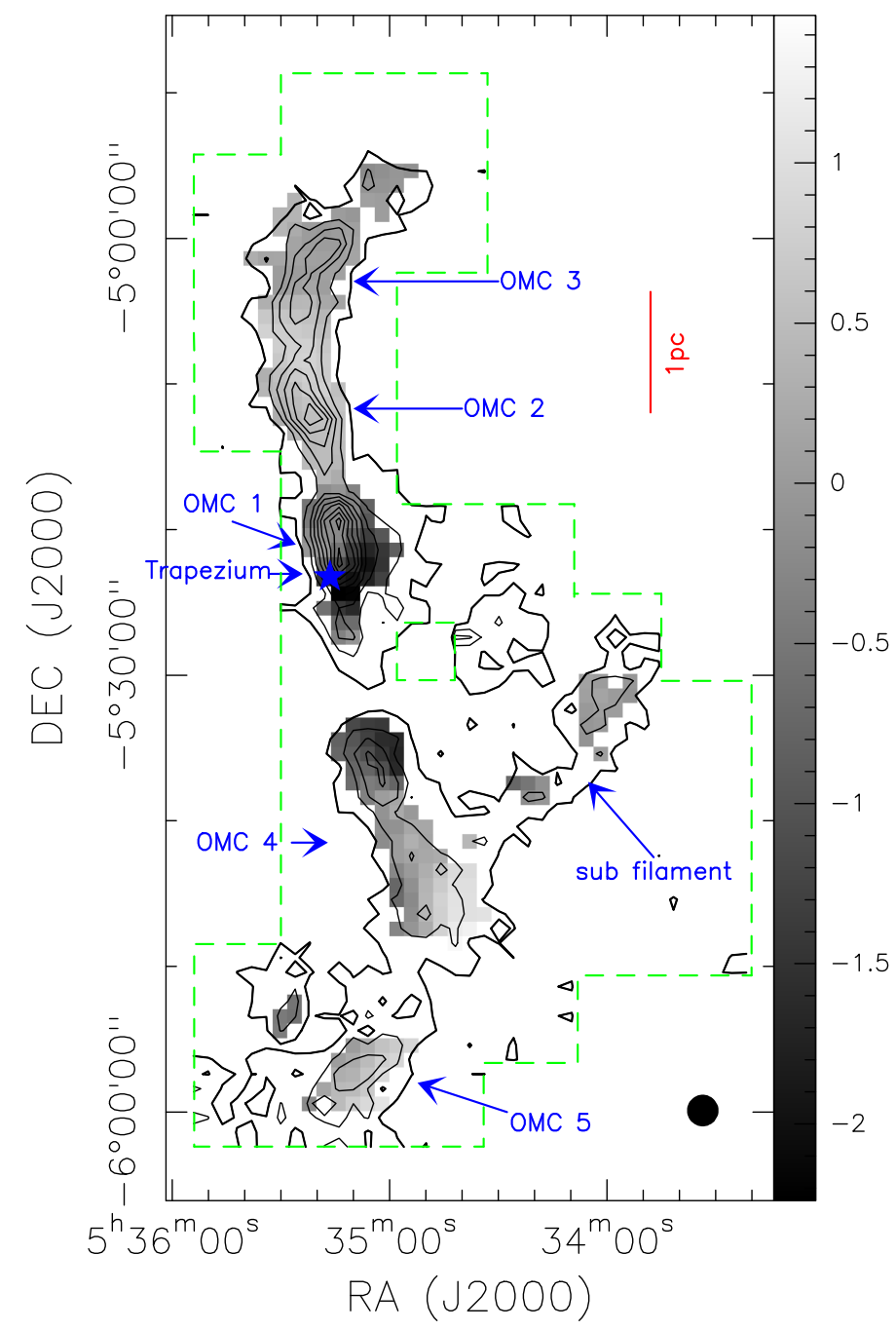

Fig. D.1. Velocity residual ( $\left.V_{\text {obs }}-V_{\text {rigid }}\right)$ map (gray image) of the ISF derived from our $\mathrm{NH}_{3}$. Contours are as in the left panel of Fig. 1.

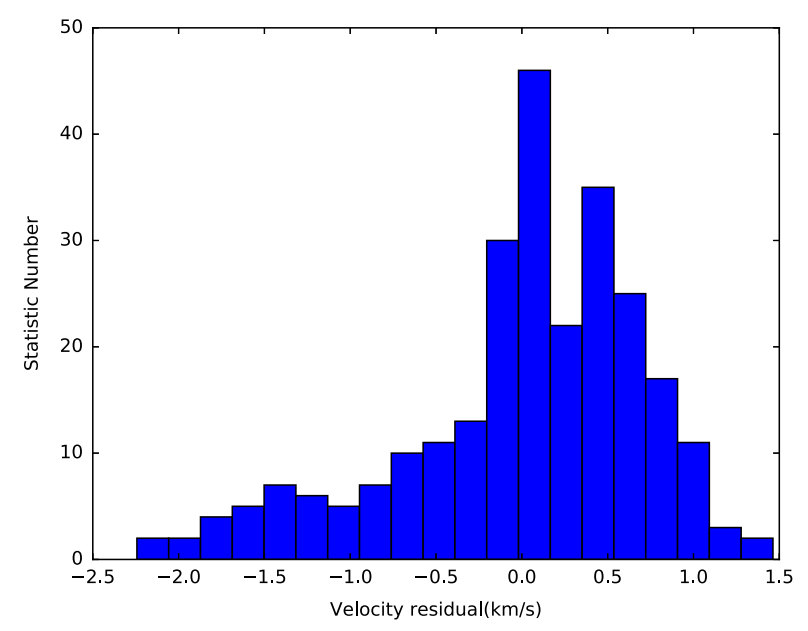

Fig. D.2. Histograms of the velocity residuals $\left(V_{\mathrm{obs}}-V_{\text {rigid }}\right)$ derived from our $\mathrm{NH}_{3}$. 